\title{
Rôle des filaments d'actine et des mécano-enzymes associées au cours de l'endocytose
}

L'endocytose est un processus crucial de la vie cellulaire qui permet l'entrée sélective au sein de la cellule de protéines ou de peptides par l'intermédiaire de récepteurs membranaires. Elle se différencie de la phagocytose par la taille des particules internalisées et de la pynocytose par la sélectivité des molécules internalisées. La phagocytose permet l'entrée à l'intérieur de la cellule de macromolécules, de bactéries ou de débris cellulaires, et de la pinocytose qui permet l'internalisation de liquide extracellulaire. La concentration des protéines internalisées est 100 à 1000 fois plus faible par pinocytose que par endocytose.

\section{Endocytose}

L'endocytose comprend plusieurs étapes qui diffèrent selon les ligands [1]. Certains sont acheminés jusqu'aux compartiments de dégradation, les lysosomes, alors que d'autres, comme la transferrine, sont recyclés à la membrane plasmique. La première étape correspondant à l'internalisation des molécules dans les endosomes précoces est commune aux deux voies. Les molécules sont ensuite séparées pour être recyclées à la membrane plasmique ou dirigées vers les lysosomes. Ces dernières sont retrouvées dans des endosomes tardifs présentant une structure multivésiculaire, puis dans les lysosomes. Les molécules recyclées sont transportées dans des structures tubulo-vésiculaires qui fusionnent avec la membrane plasmique.

L'acheminement correct des ligands dans la cellule nécessite la mise en œuvre d'un ou plusieurs mécanisme(s) de tri et de transport.

Les processus physiologiques auxquels participe l'endocytose sont multiples.
- L'endocytose permet l'absorption d'éléments nécessaires à la physiologie cellulaire. Certaines protéines présentes à l'extérieur de la cellule sont transportées vers les lysosomes pour y être transformées en petits peptides ou acides aminés qui peuvent être réutilisés par la cellule pour la biosynthèse de nouvelles protéines. - Elle permet également l'absorption d'ions ou d'atomes transportés par des protéines qui sont recyclées à la membrane plasmique sans être dégradées. Le fer, par exemple, complexé à la transferrine, est internalisé dans un compartiment d'endocytose. Au sein de ce compartiment, l'atome de fer est libéré pour être transféré dans le cytoplasme cellulaire alors que la transferrine est recyclée à la membrane plasmique. Elle pourra fixer de nouveaux atomes de fer et permettre leur internalisation.

- L'endocytose participe à la régulation des signaux transmis dans les cellules par des facteurs extracellulaires ou en réponse à des signaux extracellulaires. La liaison spécifique de facteurs de croissance à leurs récepteurs membranaires induit une cascade de signaux intracellulaires capables d'engager la cellule dans des programmes de différenciation, de prolifération, ou de motilité, etc. L'internalisation de ces molécules et de leur(s) récepteur(s) ainsi que leur transport vers un compartiment de dégradation permet d'interrompre les signaux transmis à l'intérieur de la cellule et ainsi de limiter ou contrôler la réponse aux facteurs de croissance.

Sous l'influence de différents stimulus ou dans des conditions pathologiques, certains canaux ou transporteurs, tels que les aquaporines ou le co-transporteur sodium/phosphate, sont acheminés du compartiment d'endocytose vers la membrane plasmique où ils participent aux échanges entre le milieu extérieur et la cellule. En l'absence de ces stimulus, ces protéines sont réinternalisées et stockées dans les compartiments d'endocytose. Des anomalies de ce processus d'internalisation pourraient être impliquées dans la genèse de maladies humaines. Par exemple, des délétions du domaine intracellulaire d'une des chaînes du canal sodique épithélial sont associées à une forme familiale d'hypertension artérielle (syndrome de Liddle). Ces mutations ne modifient pas l'activité de ce canal mais sont responsables de son accumulation à la membrane plasmique des cellules tubulaires rénales probablement par un défaut d'internalisation $\left(m / s n^{\circ} 6-7\right.$, vol. 12, p. 795).

- L'endocytose a également une place prépondérante dans la réponse immune et la physiopathologie de maladies systémiques [2]. En effet, certaines protéines extracellulaires internalisées puis dégradées en peptides sont présentées et chargées, dans les compartiments d'endocytose et/ou de dégradation, sur les complexes majeurs d'histocompatibilité de type II nouvellement synthétisés. Ensemble, ils sont transportés à la membrane plasmique pour être présentés aux lymphocytes T. L'organisation des compartiments d'endocytose et la régulation du transport intracellulaire des protéines internalisées vers les compartiments de dégradation sont essentielles pour permettre la rencontre des peptides et des complexes majeurs d'histocompatibilité $[2,3]$. 


\section{Rôle de l'actine}

Plusieurs auteurs ont montré que l'internalisation de récepteurs ou de protéines transmembranaires présentes à la membrane plasmique dépendait de la phosphorylation de séquences peptidiques consensuelles ou de l'ubiquitination de leur domaine intracellulaire $[1,4,5]$. L'internalisation de molécules implique également d'autres étapes comme le recrutement de récepteurs dans des domaines spécifiques de la membrane plasmique, l'invagination de celle-ci, l'élongation d'un puits membranaire, puis le resserrement du collet de ce puits pour permettre la fission de la vésicule préformée (figure 1). Ces différentes étapes nécessitent une plasticité et des changements morphologiques extrêmement dynamiques de la membrane plasmique. Depuis plus de vingt ans, il a été proposé que le cytosquelette d'actine qui sous-tend la membrane plasmique joue un rôle prépondérant dans ces processus.

Des travaux récents chez la levure et chez les eucaryotes supérieurs confirment ces hypothèses. Chez la levure, des mutations thermo-sensibles de l'actine inhibent l'entrée de ligands suggérant que l'internalisation de protéines dépend de l'intégrité des microfilaments d'actine [6]. Dans les cellules de mammifères, le groupe de van Deurs (Copenhague, Danemark) et nous-mêmes avons montré, que la désorganisation du cytosquelette d'actine par un agent dépolymérisant les filaments d'actine, la cytochalasine $\mathrm{D}$, affecte deux étapes de la voie d'endocytose : l'entrée des ligands, et leur acheminement vers les compartiments de dégradation [7, 8] (figure 2). Cependant, la dépolymérisation des filaments d'actine n'affecte pas la formation des invaginations membranaires (résultats non publiés). Les filaments d'actine pourraient participer au recrutement des récepteurs à la surface cellulaire comme le suggèrent les expériences de Sako et Kusumi (Tokyo, Japon) [9]. L'internalisation des molécules pourrait aussi être facilitée par une modification dynamique du réseau cortical d'actine engendrant une déhiscence du cytosquelette qui rendrait alors possible l'élongation de l'invagination, ou la formation de la vésicule.

L'organisation des filaments d'actine et les modifications dynamiques de cette organisation dépendent de protéines liant l'actine, ou actin binding proteins. La fimbrine, par exemple, est capable in vitro d'organiser les filaments d'actine en faisceaux. La délétion du gène sac $6 p$ qui, chez la levure, code pour un homologue de cette protéine, inhibe chez cet organisme la première étape de l'endocytose, indiquant que la fasciculation

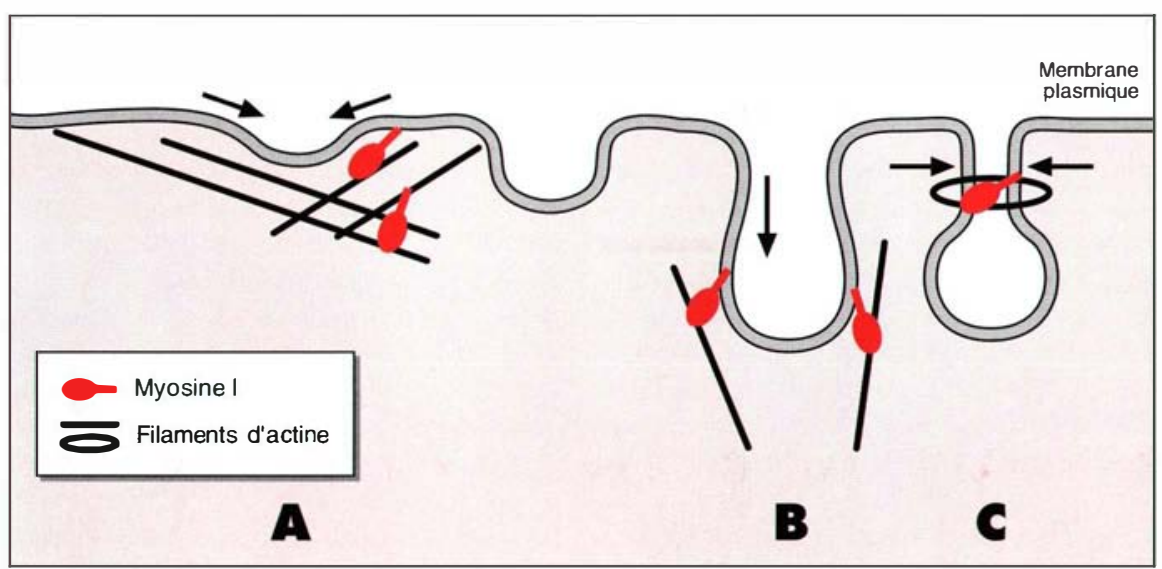

Figure 1. Différentes étapes de l'internalisation de ligands. A. Recrutement de ligands dans les invaginations membranaires; $\boldsymbol{B}$. élongation des invaginations (puits); $\boldsymbol{C}$. resserrement du collet de l'invagination puis fission. Rôles hypothétiques des filaments d'actine et des myosines I dans ces différentes

des filaments d'actine contrôlée par la protéine Sec67 est nécessaire à l'internalisation des ligands [6].

\section{Rôle des myosines I}

Très récemment, le groupe de H. Riezman (Bâle, Suisse) a également montré que l'entrée de ligands dans la levure dépendait d'une classe de mécano-enzymes associées à l'actine, les myosines I [10]. Ce groupe de protéines interagit par son domaine carboxy-terminal avec la membrane plasmique et, par son domaine amino-terminal ou domaine moteur, avec les filaments d'actine. Les myosines ont la propriété d'exercer, en présence d'ATP, une force mécanique permettant in vitro le déplacement de billes de latex ou de vésicules membranaires [11]. In vivo, elles pourraient exercer des forces mécaniques sur la mem-

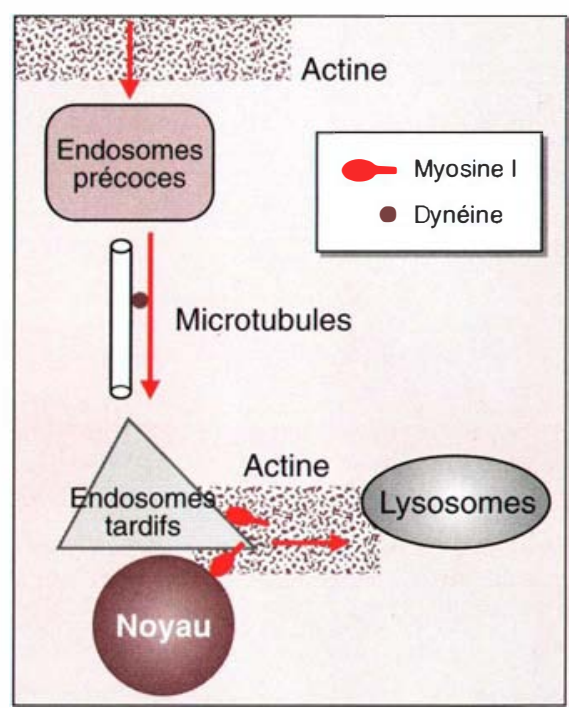

Figure 2. Implication des filaments d'actine et des myosines I le long de la voie d'endocytose dans les cellules eucaryotes supérieures. Les filaments $d^{\prime}$ actine sont impliqués dans deux étapes: pour l'internalisation des ligands et pour leur transfert des endosomes tardifs vers les lysosomes. Les myosines I sont impliquées dans cette dernière étape. Les microtubules et une mécano-enzyme associée, la dynéine, facilitent le transport des ligands des endosomes précoces vers les endosomes tardifs. 
brane plasmique en s'ancrant au cytosquelette d'actine. Elles pourraient participer ainsi aux différents mécanismes d'endocytose proposés ci-dessus (invaginations membranaires, recrutement de ligands au sein des puits d'invagination, fission des vésicules de la membrane plasmique) [12] (figure 1).

Plusieurs de ces myosines ont été identifiées dans des cellules d'eucaryotes supérieurs. Certaines, comme la myosine I de la bordure en brosse, sont synthétisées spécifiquement dans certains types cellulaires alors que d'autres sont ubiquitaires. La production de la myosine I de la bordure en brosse, tronquée du domaine responsable de l'activité mécano-enzymatique, entraîne une fragmentation du compartiment d'endocytose dans plusieurs types cellulaires. Elle modifie égalcment la quantité de ligands délivrés aux lysosomes, alors qu'elle ne modifie pas leur cinćtique d'entrée ni le recyclage de la transferrine à la membrane plasmique [13]. Ces résultats suggèrent que, dans les cellules eucaryotes, une myosine I pourrait régler la deuxième étape de la voie d'endocytose, dans laquelle l'actine intervient (figure 2).

Les mécanismes impliqués dans la régulation de l'activité des myosines I ne sont pas encore parfaitement connus. Cependant, toutes ces molécules possèdent plusieurs sites de liaison de la calmoduline. Pour certaines d'entre elles, il a pu être montré in vitro que le calcium et la calmoduline règlent leurs propriétés mécano-enzymatiques. Cela suggère que, in vivo, les variations de la concentration de calcium en réponse à des signaux intra- ou extracellulaires pourraient également contrôler la fonction de ces myosines.

Plusieurs étapes de la voie d'endocytose dépendent, par conséquent, du cytosquelette. On a précédemment observé que les microtubules et des mécano-enzymes associées (les dynéines) facilitent le passage de ligands des endosomes précoces aux endosomes tardifs. De la même manière, les filaments d'actine pourraient, en association à des mécanoenzymes, les myosines I, régler le transport de vésicules d'endocytose. Ils pourraient également être impliqués dans le recrutement de ligands dans des vésicules en formation ou dans la fission de ces vésicules de la membrane plasmique.

La mise en évidence de nouveaux mécanismes moléculaires impliqués dans la régulation de l'endocytose devrait permettre, dans l'avenir, de mieux disséquer et de mieux comprendre les différentes étapes du trafic le long de la voie d'endocytose et ses conséquences physiologiques ou physiopathologiques

\section{Antoine Dürrbach}

Chef de clinique, Inserm U.426, service de néphrologie, Hôpital Bichat-ClaudeBernard, 46, rue Henri-Huchard, 75018 Paris, France.

\section{Évelyne Coudrier}

Directeur de recherches au Cnrs, Cnrs UMR 144, Institut Curie, 26, rue d'Llm, 75005 Paris, France.

\section{TIRÉS À PART}

A. Dürrbach.

\section{RÉFÉRENCES}

1. Carillo S, Pariat M, Jariel-Encontre 1, Steff A, Piechaczyk M. Le catabolisme protéique intracellulaire: une fonction biologique majeure. Partie 1 : les mécanismes de dégradation. méderine/sciences $1995 ; 11: 723-34$
2. Carillo S, Pariat M, Jariel-Encontre I, SteffA, Lorca T, Piechaczyk M. Le catabolisme protéique intracellulaire : une fonction biologique majeure. Partie II: exemples de dégradation conditionnelle et genèse des peptides antigéniques. médecine/sciences 1995; $11: 845-52$.

3. Rabourdin-Combe C, Bertolino P, CalinLaurens V, Gerlier D. La présentation de l'antigène aux lymphocytes T. médecine/ sciences $1991 ; 7: 674-80$.

4. Matter $\mathrm{K}$, Whitney JA, Yamamoto EM, Mellman 1. Common signals control low density lipoprotein receptor sorting in endosomes and the Golgi complex of MDCK cells. Cell $1993 ; 74: 1053-64$.

5. Hicke L, Riezman H. Ubiquitination of a yeast plasma membrane receptor signals its ligand-stimulated endocytosis. Cell 1996; 84: 277-87.

6. Kübler E, Riezman H. Actin and fimbrin are required for the internalization step of endocytosis in yeast. $E M B O J 1993 ; 12$ : 2855-62.

7. Dürrbach A, Louvard D, Coudrier E. Actin filaments facilitate two steps of endocytosis. J Cell Sci 1996; 109 : 457-65.

8. van Deurs B, Holm PK, Kayser L, Sandvig $\mathrm{K}$. Delivery to lysosomes in human carcinoma cell line HEp-2 involves an actin filament-facilitated fusion between mature endosomes and preexisting lysosomes. Eur I Cell Biol 1995 ; 66 : 309-23.

9. Sako Y, Kusumi A. Compartmentalized structure of the plasma membrane for receptor movements as revealed by a nanometer-level motion analysis. J Cell Biol 1994 ; $125: 1251-64$

10. Gelli MI, Riezman H. Role of type I myosins in receptor-mediated endocytosis in yeast. Science 1996 ; 272 : 533-5.

11. Eldin P, Cornillon B, Mornet D, Léger J. Une nouvelle jeunesse pour les myosines. médecine/sciences $1995 ; 11$ : 1005-16.

12. Coudrier E, Dürrbach A, Louvard D. Do unconventional myosins exert functions in dynamics of membrane compartments ? FEBS Lett 1992 ; 307 : 87-92.

13. Dürrbach $\mathrm{A}$, Collins $\mathrm{K}$, Matsudaira $\mathrm{P}$, Louvard D, Coudrier E. Brush border myosin-1 truncated in the motor domain impairs the distribution and the function of endocytic compartments in an hepatoma cell line. Proc Natl Acad Sci USA 1996; 93. 7053-8.

\section{GÉNÉTIQUE DE LA FERTILITÉ MASCULINE - Collioure, France - 4-6 septembre 1997 JEUDI 4 SEPTEMBRE \\ Contrôle génétique de la sperrnatogenèse \\ C. Sultan (France), N. Hecht (USA), D. Page (USA), A. Spira (France), \\ C. Gagnon (Canada), P.N. Schlegel (USA), A. Chandley (UK), H. Tournaye (Belgique) \\ VENDREDI 5 SEPTEMBRE \\ Génétique du spermatozoïde \\ S. Ward (USA), R.H. Martin (Canada), J.D. Schulman (USA), D. Sakkas (Suisse) \\ Session de posters \\ Table ronde d'éthique \\ M. Donough (USA), M. Serres (France), F. Collins (USA), A. Kahn (France) \\ SAMEDI 6 SEPTEMBRE \\ Rôle du spernnatozoïde dans l'embryogenèse \\ L. Janny (France), G. Schatten (USA), N. De Groot (Pays-Bas), J. Cummins (Australie) Secrétariat du congrès : Hélène Moutaffian, CHU la Grave, Laboratoire de FTV 31052 ToulouseCedex, France - Tél. : (33) 61.77.78.58 - Fax : (33) 61.59.24.83}

